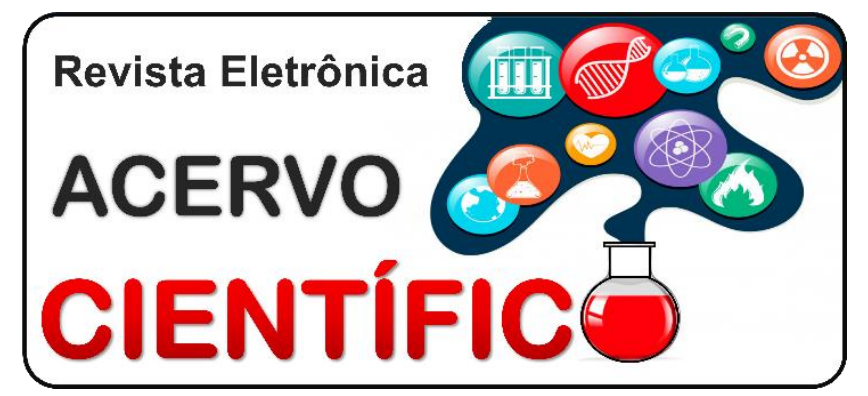

\section{REVISÃO BIBLIOGRÁFICA}

Recebido em: 4/2020

Aceito em: $4 / 2020$

Publicado em: 6/2020

\title{
Motivação para a aprendizagem em crianças escolares: revisão narrativa
}

\author{
Motivation for learning in school children: narrative review
}

Motivación para el aprendizaje en escolares: revisión narrativa

Leomir Barros Coutinho de Melo ${ }^{1,4 *}$, Marinaldo Alves de Souza ${ }^{2,4}$, Jaiurte Gomes Martins da Silva ${ }^{3,4}$.

\begin{abstract}
Resumo: Esse artigo buscou discorrer sobre a motivação para a aprendizagem em crianças escolares. A motivação relacionada ao processo educacional tem sido alvo de muitas pesquisas nacionais e internacionais, principalmente com o decorrer dos anos. Isto, porque, esta é uma relevante variável a ser investigada quando se trata do processo de ensino aprendizagem, pois, sua ausência tem sido atribuída a uma queda de investimento pessoal do estudante na realização das tarefas solicitadas pelo professor, pouca participação nas aulas, pouco tempo dedicado aos estudos e possível afastamento do processo de aprendizagem. Ou seja, a motivação é fundamental na vida dos estudantes por interferir no comportamento dos estudantes frente às questões educacionais, e, por isso, tem sido tão discutida na literatura nacional e internacional. A motivação é uma variável de grande importância por exercer influência no comportamento dos estudantes diante dos desafios a eles propostos, e deste modo, afetar o processo de aprendizagem deles.
\end{abstract}

Palavras-chave: Educação, Desmotivação, Ensino.

Abstract: This article sought to discuss the motivation for learning in school children. Motivation related to the educational process has been the subject of many national and international researches, mainly over the years. This, because, this is a relevant variable to be investigated when it comes to the teaching-learning process, because its absence has been attributed to a drop in the student's personal investment in performing the tasks requested by the teacher, little participation in classes, little time dedicated to studies and possible departure from the learning process. In other words, motivation is fundamental in the students 'lives because it interferes with the students' behavior regarding educational issues, and, for this reason, it has been so discussed in national and international literature. Motivation is a variable of great importance because it influences the students' behavior in the face of the challenges proposed to them, and in this way, affects their learning process.

Keywords: Education, Demotivation, Teaching.

1Universidade Federal de Pernambuco (UFPE), Recife - PE. *E-mail: leomirbcm3@gmail.com

2Universidade de Pernambuco (UPE), Nazaré da Mata - PE.

${ }^{3}$ Universidade Federal Rural de Pernambuco (UFRPE), Recife - PE.

${ }^{4}$ Faculdade Santíssima Trindade (FAST), Nazaré da Mata - PE. 
Resumen: Este artículo buscaba discutir la motivación para el aprendizaje en escolares. La motivación relacionada con el proceso educativo ha sido objeto de muchas investigaciones nacionales e internacionales, principalmente a lo largo de los años. Esto, porque, esta es una variable relevante para investigar cuando se trata del proceso de enseñanza-aprendizaje, ya que su ausencia se ha atribuido a una caída en la inversión personal del estudiante para llevar a cabo las tareas solicitadas por el maestro, poca participación en las clases, poca Tiempo dedicado a los estudios y posible desviación del proceso de aprendizaje. En otras palabras, la motivación es fundamental en la vida de los estudiantes porque interfiere con el comportamiento de los estudiantes con respecto a cuestiones educativas y, por esta razón, se ha discutido en la literatura nacional e internacional. La motivación es una variable de gran importancia porque influye en el comportamiento de los estudiantes frente a los desafíos que se les proponen y, de esta manera, afecta su proceso de aprendizaje.

Palabras clave: Educación, Desmotivación, Enseñanza.

\section{INTRODUÇÃO}

A educação nem sempre é circundada apenas por aprovações e sucessos. No transcorrer do ensino, muitas vezes, nos deparamos com transtornos que deixam os estudantes paralisados perante o processo de aprendizagem. Na maior parte dos casos, é o docente o primeiro a reconhecer que a criança está com alguma dificuldade. E esta dificuldade pode ser a desmotivação da criança, que interfere negativamente no processo de ensino-aprendizagem (TABILE AF e JACOMETO MCD, 2017).

A ausência de motivação se transformou num transtorno na educação, visto que a sua falta simboliza diminuição no investimento pessoal de qualidade nas atividades de aprendizagem, que pode induzir a saída escolar. No contexto escolar, a motivação para o aprendizado, é um tema que admite a discussão não apenas na perspectiva dos estudantes, mas, sobretudo do ponto de vista do docente. Ambos, quando estão motivados, esforçam-se mais na execução das tarefas escolares (CAMARGO CACM, 2019).

Permanecer motivado a continuar e enfrentar as dificuldades que surgem no transcorrer do processo escolar é somente um dos problemas a ser encarado. Tem sido dada grande atenção a motivação, visto que ela é analisada de relevância fundamental na vida dos indivíduos e no desempenho escolar dos estudantes (MARTINELLI SC e SISTO FF, 2011). São diversas as condições que podem gerar influência na motivação, podendo ser fatores internos ao indivíduo, e o ambiente escolar (MARTINELLI SC e SISTO FF, 2011) até a família (GUIDETTI AR, 2013).

A motivação é entendida como um dos fatores imprescindíveis e essenciais para o progresso do ser humano, de maneira especial das capacidades sociais e cognitivas e das habilidades, pois o processo de aprender não é inerte, pelo contrário, está em continua inovação e evolução, acima de tudo, deve ser motivado. Com referência a motivação, Piletti N (2013), afirma que a motivação é agente indispensável da aprendizagem. É possível existir aprendizagem sem docente, sem estabelecimento de ensino, sem computador, sem livro e sem muitos e diversos recursos. Mas, se não existir motivação, não existirá aprendizagem, ainda que haja todos esses recursos adequados.

As pesquisas de Accorsi DMP, et al. (2007), Martini ML, et al. (2004) e Goya A, et al. (2008) destacam que, no contexto dos estudantes, a desmotivação para o aprendizado pode resultar em um baixo rendimento escolar, tendo em vista a insuficiente aplicação no próprio aprendizado. Os educandos desmotivados pelas atividades escolares exibem desempenho aquém de suas reais capacidades, não participam das aulas, estudam pouco ou nada, se distraem facilmente e afastam-se do processo de aprendizagem. Com isso, aprende pouco correndo o risco de evadir da escola restringindo suas chances vindouras (BZUNECK JA, 2004).

Quando estamos desmotivados, ficamos indisposto para desempenhar tarefas, por mais significativas que sejam. Esse ímpeto de estarmos motivados depende de vários elementos que podem afetar o processo de aprendizagem, como o entendimento de sucesso frente à aprendizagem, o caráter mais ou menos 
expressivo que a disciplina apresenta para o discente e as suas expectativas, o nível de preocupação dos educandos diante de determinada tarefa, a maneira como os docentes executam o discurso no contexto sala de aula, entre outros. A motivação dos discentes é uma constante preocupação a todos os indivíduos envolvidos diariamente no processo de aprendizagem, independentemente dos conteúdos curriculares da disciplina que lecionam ou da metodologia usada pelo professor (RAMOS VAB, 2019).

Segundo Engelmann E (2010); diversos fatores estão envolvidos quando se refere à motivação, e ela está envolvida em vários fatores que instigam o indivíduo a alcançar certo comportamento perante uma circunstância/problema. Um estudante quando está motivado consegue alcançar melhor capacidade de atenção/concentração e persistência nas atividades escolares, executando suas obrigações escolares e deste modo o satisfazendo quando alcança os desígnios estabelecidos.

Alcará AR e Guimarães SER (2007); afirmam que um estudante motivado está inteiramente comprometido com o processo de aprendizagem, e isto trás efeitos positivos que o leva a buscarem novos conhecimentos, participando nas atividades com entusiasmo e demonstrando disposição para novas oportunidades e desafios. Em suma, as experiências escolares dos estudantes podem gerar influência de forma direta ou indireta na motivação, autoconceito, e responsabilidade nas atividades da escola e rendimento (JANOSZ M, et al., 1997 apud ARCHAMBAULT I, 2007).

Diante da importância da motivação para o processo de ensino-aprendizagem, o trabalho tem por objetivo discorrer sobre a motivação para a aprendizagem em crianças escolares através de uma revisão narrativa do tema.

\section{REVISÃO BIBLIOGRÁFICA}

Em ligação com a aprendizagem dos estudantes, a escola vem sempre procurando aperfeiçoar os apontadores de ensino e aprendizagem, procurando aprimoramento, atualização e formação dos docentes. Enfatiza-se que a instituição escolar estimula a cada vez mais comprometimento e acompanhamento por parte dos pais com as atividades diárias e tarefas dos seus filhos, e ainda, procura-se mais abarcamento e ativa participação da família na educação; assistência as tarefas escolares, valores éticos, morais e limites (REQUIA R, 2015). Porém, muitas instituições têm sofrido com estudantes desmotivados e desinteressados considerando a forma de como se dá o processo educacional.

O termo motivação resulta do verbo latim "movere", que apresenta a idéia de movimento (SIQUEIRA LGG e WECHSLER SM, 2006). Esta ideia acha-se presente em muitas definições, visto que a motivação induz um indivíduo realizar algo, persistindo na ação e ajudando-o a concluir a atividade (SANTROCK JW, 2009).

Nas últimas décadas averígua-se uma retomada das pesquisas sobre motivação associada ao ambiente escolar. Estas pesquisas têm mostrado que as crianças estão chegando desmotivadas nas escolas. Isso pode ser um fator que pode gerar a evasão escolar (KNÜPPE L, 2006). Segundo os autores Jesus S e Abreu M (1993); a ausência de motivação dos estudantes pode ser caracterizada por meio de determinados apontadores tais como: débil comprometimento no desempenho das suas atividades, redução da participação dos estudantes nas aulas e, ainda, uma diminuição marcante do tempo expendido pelos estudantes a estudar. Logo, o sucesso escolar, irá receber influências destes fatores de desmotivação.

Educadores e professores têm demonstrado sua inquietação no que diz respeito à motivação e o desempenho escolar dos estudantes. Tem-se assegurado que um estudante motivado exibe quando comparado aos demais, um melhor desempenho, em consequência do investimento exclusivo que aplica na atividade que executa. Contudo, pesquisas chegaram à conclusão que a afinidade entre motivação e aprendizagem não se limita a uma pré-condição da primeira para o acontecimento da segunda, no entanto existe uma afinidade de reciprocidade entre elas (MITCHELL JV, 1992; SCHUNK DH, 1991).

Dessa maneira, a motivação é eficiente provocador para um bom resultado no desempenho e na aprendizagem, bem como a aprendizagem pode intervir na motivação (MITCHELL JV, 1992; NETO, S P, 1987). De acordo com Lourenço AA e De Paiva MOA (2010); constata-se que há uma afinidade entre a 
motivação e aprendizagem, averiguando uma relação de mutualidade entre ambas. Em termos educativos, um estudante encontra-se disposto para aprender autonomamente quando está motivado (PEREIRA MLN, 2010)

A maioria das contribuições é resultado da teoria da autodeterminação, sugerida por Deci e Ryan (DECl EL e RYAN RM, 1985). Este modelo teórico tem situado suas pesquisas nas situações sociocontextuais que enfraquecem ou facilitam a automotivação e desenvolvimento saudável, que são os processos naturais.

Do ponto de vista teórico da Teoria da Autodeterminação, para que ocorra a promoção de processos naturais de motivação devem-se considerar fundamentais as três necessidades psicológicas básicas que são: autonomia, competência e de estabelecimento ou pertencimento de vínculos que, quando são satisfeitas, promovem a saúde mental e maior motivação. Assim sendo o estudo destas necessidades tem sido fonte de interesse das variadas áreas do conhecimento como a educação, psicoterapia e saúde, entre outras (RYAN RM e DECI EL, 2000).

\section{Motivação intrínseca e extrínseca}

De acordo com a Teoria da Autodeterminação (1985), os indivíduos são motivados por duas principais orientações; a intrínseca e a extrínseca. E neste modelo teórico, que explica o processo motivacional, a motivação extrínseca apresenta algumas regulações, que são: regulação externa, regulação introjetada, regulação identificada e regulação integrada, e por fim a motivação (DECI EL e RYAN RM, 1985).

A motivação intrínseca decorre na participação do educando nas tarefas escolares sem o controle de nenhum fator externo, sejam pressões, ameaças, recompensas ou outros (RUFINI SE, et al., 2011). Nela o processo assinalado pela escolha pessoal, prazer e satisfação (Brickell TA e Chatzisarantis NLD, 2007) e de acordo com Deci EL E Ryan RM (2000) não há a necessidade de recompensa externa ou reforço. Quando o sujeito é intrinsecamente motivado, ele entra na atividade por vontade própria, conta-se pela satisfação do processo de conhecê-la, explorá-la, aprofundá-la ou pelo prazer. Procedimentos intrinsecamente motivados são frequentemente associados com o interesse, bem-estar psicológico, persistência e alegria

Na regulação externa, de acordo com Ryan RM e Deci EL (2000), acontece quando o procedimento é gerido por premiações externas ou medo de conseqüências negativas como apreciações sociais. A pessoa simplesmente atende a controladores externos, como no caso de fazer uma tarefa por mera obediência, ou visando alguma recompensa, ou para evitar punições ou por pressões externas (RUFINI SE, et al., 2011).

Na regulação introjetada acontece quando o procedimento sofre regulação de uma fonte de motivação que, no início externa, é internalizada, como procedimentos que são reforçados por pressões internas como a culpa ou a necessidade de ser aceito (RYAN RM e DECI EL, 2000). A pessoa é motivada a comportar-se de uma determinada forma para atender as instâncias ligadas a autoestima ou impedir sentimento de ansiedade

A regulação identificada ocorre quando a pessoa avaliar certo comportamento como de importância pessoal, permitindo sua regulação como própria (RUFINI SE, et al., 2011). Nela o indivíduo executa uma tarefa à qual ele não pode escolher, ou seja, uma atividade que é analisada como relevante a ser executada, ainda que não the seja interessante.

Ryan RM e Deci EL (2000) destacam que os processos reguladores associados à motivação extrínseca identificada são: Importância pessoal e a valorização consciente. Um exemplo bom é de um jovem que tem como objetivo de vida tornar-se escritor e, por isso, admite como valor próprio praticar redação e leitura (RUFINI SE, et al., 2011).

Na regulação integrada, é aquela que se define pelo comportamento manifestado por escolha pessoal, sem coação e com completa autonomia, considerado como alguma coisa pessoalmente relevante, porque incorporado ao próprio self (RUFINI SE, et al., 2011). Este tipo de regulação está bastante próximo da própria motivação intrínseca, e se diferencia por ocorrer à liberdade de escolha, finalizar o componente específico de interesse pela atividade e com componente afetivo (RYAN RM e DECI EL, 2000). Na 
motivação extrínseca por regulação integrada, mesmo sendo apesar ser tida com um grau relativo de autonomia, existe a precisão de um elemento externo que dá ímpeto e sentido a ação. Por exemplo, se a meta de um estudante de mestrado for produzir sua dissertação com qualidade pode ser integrada aos seus valores pessoais, de tal maneira que ele não diferencie com facilidade as condições externas das suas próprias condições. Contudo, se o diploma de mestre não estivesse mais ligado a apresentação do trabalho ele possivelmente não continuaria na ação (RUFINI SE, et al., 2011).

A falta de motivação, segundo Ryan RM e Deci EL (2000), é uma constituição motivacional encontrada em pessoas que ainda não estão apropriadamente habilitadas a identificar um bom motivo para realizar certo comportamento, não existe qualquer tipo de regulação, seja interna ou externa. Deci EL e Ryan RM (2000), também consideram que, ainda que a motivação intrínseca consista em um tipo de motivação importante, ela não é o único tipo de motivação autodeterminada e que, à proporção que nos desenvolvemos a maior parte das coisas que exercemos não são motivadas exclusivamente de maneira intrínseca. A despeito dessas contestações os dois modelos motivacionais vêm ao longo dos anos sendo estudados, pela compreensão que essa variável apresenta-se como auxiliadora à aprendizagem. Ainda não se conhece como a escola colabora para as modificações acontecidas nas orientações motivacionais dos educandos, ainda que venha se averiguando um significativo e progressivo declínio na motivação intrínseca dos mesmos no ensino médio e no decorrer do ensino fundamental (MARTINELLI SC, 2014).

Estudo feito por Lepper MR, et al. (2005), que tinha como um dos objetivos averiguar no transcorrer do período escolar, a relação entre a motivação intrínseca e extrínseca, por meio de medidas que independem destes dois construtos confirmaram esses resultados. Foi encontrado que a motivação intrínseca diminui com o passar das series (3ำ para $8^{\circ}$ série) nas escolas americanas. O mesmo resultado foi encontrado por Otis N, et al., (2005), que se comprometeram em uma amostra com estudantes explorar as modificações nas orientações motivacionais. Nesta pesquisa observou-se que tanto a motivação intrínseca quanto a extrínseca diminuía com o avançar das series.

Em outra pesquisa Corpus $\mathrm{JH}$, et al. (2009) verificaram a característica e as mudanças na motivação de alunos durante o decorrer de um ano escolar em estudantes do $3^{\circ}$ ano ao $8^{\circ}$ ano. As análises exibiram variações nas orientações motivacionais extrínsecas e intrínsecas dos educandos durante 0 ano de escolarização. O declínio na motivação intrínseca foi mais confessado pelos adolescentes que os alunos do ensino fundamental, que tiveram uma redução na motivação extrínseca. Houve influência mútua de maneira recíproca e positiva na motivação intrínseca e as atividades realizadas em sala de aula. E, por outro lado, comportamentos mais pobres em sala de aula pressagiaram minimamente elevados níveis de motivação extrínseca, ainda que este tipo de motivação não tenha sido indicado como promotor de notas mais baixas.

A principal finalidade de Gillet N, et al. (2012) foram pesquisar a falta de motivação e a motivação intrínseca e extrínseca em uma amostra que apresentava 1600 estudantes com idades entre 9 a 17 anos do ensino fundamental e médio. Os resultados expuseram uma diminuição sistemática da motivação extrínseca autodeterminada e da motivação intrínseca dos 9 aos 12 anos de idade, havendo uma lenta estabilidade aos 15 anos e após esse ponto acontece um acréscimo. Em segundo lugar, houve um declínio até os 12 anos de idade da motivação extrínseca não autodeterminada, e após este ponto a mesma consolidou-se. Finalmente, a motivação foi relativamente constante e baixa dos 9 até os 17 anos.

O aproveitamento do estudante e a motivação diante as aprendizagens escolares vem sendo tema de pesquisas a décadas, e em vários países, outras pesquisas têm sido conduzidas. Estas pesquisas vêm indicando a relevância da motivação para a aprendizagem e o desempenho dos alunos, como pesquisado no trabalho dirigido por Gottfried AE, et al., (2007), que investigou a motivação intrínseca para matemática acadêmica e o desempenho em matemática, em um estudo longitudinal, com estudantes de idades entre 9 a 17 anos. Em média, com o passar dos anos tanto a motivação quanto o desempenho na matéria (matemática) decaíram, desde o período infantil até a adolescência.

No Brasil, foram dirigidas várias pesquisas na perspectiva da teoria da autodeterminação e que tinham por objetivo constatar como a motivação se aloca no percurso do processo de escolarização (NEVES ERC e BORUCHOVITCH E, 2004; HIGA SL e MARTINELLI SC, 2006; MARTINELLI SC, 2008; MARTINELLI SC 
e SISTO FF, 2010). Com ressalva da pesquisa de Neves ERC e BoruchovitcH E, (2004), os outros pesquisadores encontraram um atraso na motivação com o aumento da escolaridade, ou seja, quanto mais avançado o aluno fica menor sua desmotivação, ratificando determinados achados da literatura internacional.

Costa GDF (2005) dirigiu um trabalho que tinha como finalidade avaliar as relações entre o desempenho escolar e motivação. A amostra foi composta por 127 educandos do 8a ano, com idades entre 11 e 12 anos, em relação a disciplina matemática (resoluções de equação de primeiro grau). Os resultados mostraram que os discentes expuseram uma disposição à orientação motivacional dominantemente intrínseca. Ainda assinalaram que os discentes enfatizaram o aproveitamento do estudo e os privilégios deste como valor em si mesmo, ressaltando a novidade, curiosidade e esforço como relevantes para a aprendizagem. Os dados sugeriram também que discentes que estudavam apenas para aprender exibiram desempenho melhor na prova de matemática em afinidade aos outros discentes.

Paiva e Boruchovitch (2010) igualmente se propuseram a pesquisar, entre outras variáveis, o desempenho acadêmico e as orientações motivacionais de estudantes do $4^{\circ}$ e do $6^{0}$ ano do ensino fundamental. Quanto à motivação, as autoras verificaram que a maior parte dos estudantes que tomou parte da pesquisa exibiu uma orientação motivacional dominantemente intrínseca $(77,5 \%)$, ainda que tenham notado um atraso da mesma com o passar dos anos. Além disso, abalizam para a preponderância da motivação intrínseca em estudantes com positivo desempenho.

Sá (2004) após realizar sua pesquisa, afirma que um discente motivado considera como parte significante e relevante do seu projeto de vida e impõe um significado positivo e pessoal à própria aprendizagem, faz continuas avaliações se têm um jeito especial para uma determinada, se é mau ou bom discente e avalia o valor que estes resultados têm para os outros e para si.

\section{CONSIDERAÇÕES FINAIS}

Ainda há muito que investigar no que diz respeito à motivação e o processo de ensino aprendizagem. 0 presente estudo procurou expor a motivação para a aprendizagem e mostrou que esta variável (motivação) gera interferência no desempenho escolar dos discentes, tais como demonstrar persistência, despender esforços, prestar atenção entre outros. Diversos estudos concluíram que esta variável é de grande importância e precisa ser investigada quando se refere ao processo de ensino aprendizagem, pois pode interferir no mesmo. É necessária ainda, a investigação do tema abordado ao longo deste artigo, usando abordagens qualitativas e quantitativas, para aprofundamentos nos fatores que possam gerar influência na motivação dos estudantes.

\section{REFERÊNCIAS}

1. CAMARGO CACM, et al. A importância da motivação no processo ensino-aprendizagem. Revista Thema, 2019; 16(3): 598-606.

2. COSTA GDF. Relações entre as orientações motivacionais e o desempenho escolar de alunos da $7^{\mathrm{a}}$ série do ensino fundamental em matemática, na resolução de equações do 1ำ grau. 2005.

3. DECI EL e RYAN RM. The "What" and "Why" of Goal Pursuits: Human Needs and the Self-Determination of Behavior. Psychological Inquiry, 2000; 11(4): 37-41.

4. DECI EL e RYAN RM. Intrinsic motivation and self-determination in human behavior. New York and London: Plenum, 1985; 86.

5. HIGA SL e MARTINELLI SC. As orientações motivacionais de estudantes do ensino fundamental. Teoria e Prática da Educação, 2006; 9(2): 169-177.

6. LOURENÇO AA e PAIVA MOA. A motivação escolar e o processo de aprendizagem. Ciências \&Cognição, 2010; 15(2).

7. MARTINELLI SC. A study on the motivation among elementary school children. In: Anais do V Congreso Internacional de Psicología y Educación. 2008.

8. MARTINELLI SC e SISTO FF. Motivação de estudantes: um estudo com crianças do ensino fundamental. Avaliação Psicologica: Interamerican Journal of 5 Psychological Assessment, 2010; 9(3): 413-420. 
9. MARTINELLI SC. Um estudo sobre desempenho escolar e motivação de crianças. Educar em revista, 2014; 53: 201-216.

10. MITCHELL JV. Interrelationships and predictive efficacy for indices of intrinsic, extrinsic, and self-assessed motivation for learning. Journal of Research \& Development in Education, 1992.

11. NEVES ERC e BORUCHOVITCH E. A motivação de alunos no contexto da progressão continuada. Psicologia: teoria e pesquisa, 2004; 20(1): 77-85.

12. OTIS N, et al. Latent motivational change in an academic Setting: A 3-Year longitudinal study. Journal of educational psychology, 2005; 97(2): 170.

13. PAIVA MLMF e BORUCHOVITC E. Orientações motivacionais, crenças educacionais e desempenho escolar de estudantes do ensino fundamental. Psicologia em Estudo, 2010; 15(2): 381-389.

14. PEREIRA MLN. Factores que favorecen el desarrollo de una actitud positiva hacia las actividades académicas. Revista educación, 2010; 34(1): 31-53.

15. RAMOS, Vera Alexandra Barbosa. A motivação e o sucesso escolar, 2019.

16. REQUIA R. A relação entre a motivação e desempenho escolar em alunos dos anos iniciais do ensino fundamental: um estudo na Escola Municipal de Ensino Fundamental Padre Gabriel Bolzan. 2015.

17. RUFINI SE, et al. Estudo de validação de uma medida de avaliação da motivação para alunos do ensino fundamental. Psico-USF, 2011; 16(1): 1-9.

18. RYAN RM e DECI EL. Intrinsic and extrinsic motivations: Classic definitions and new directions. Contemporary educational psychology, 2000; 25(1): 54-67.

19. SCHUNK DH. Self-efficacy and academic motivation. Educational psychologist, 1991; 26(3-4): 207-231.

20. TABILE AF e JACOMETO MCD. Fatores influenciadores no processo de aprendizagem: um estudo de caso. Revista Psicopedagogia, 2017; 34(103): 75-86. 